\title{
A COMPUTATIONAL FLUID DYNAMICS STUDY OF SWIRLING FLOW REDUCTION BY
}

\section{USING ANTI-VORTEX BAFFLE}

\author{
H. Q. Yang, \\ CFD Research Corp./Jacob ESSSA Group \\ Huntsville, AL 35805 \\ and \\ John W. Peugeot and Jeff S. West \\ Fluid Dynamics Branch-ER42 \\ George C. Marshall Space Flight Center \\ MSFC, AL 35812
}

An Extended Abstract submitted to

\begin{abstract}
49th AIAA/ASME/SAE/ASEE Joint Propulsion Conference \& Exhibit and 11th International Energy Conversion Engineering Conference
\end{abstract}

15 - 17 July 2013

San Jose, California

\begin{abstract}
An anti-vortex baffle is a liquid propellant management device placed adjacent to an outlet of the propellant tank. Its purpose is to substantially reduce or eliminate the formation of free surface dip and vortex, as well as prevent vapor ingestion into the outlet, as the liquid drains out through the flight. To design an effective anti-vortex baffle, Computational Fluid Dynamic (CFD) simulations were undertaken for the NASA Ares I vehicle LOX tank subjected to the simulated flight loads with and without the anti-vortex baffle. The Six Degree-Of-Freedom (6DOF) dynamics experienced by the Crew Launch Vehicle (CLV) during ascent were modeled by modifying the momentum equations in a CFD code to accommodate the extra body forces from the maneuvering in a non-inertial frame. The present analysis found that due to large moments, the CLV maneuvering has significant impact on the vortical flow generation inside the tank. Roll maneuvering and side loading due to pitch and yaw are shown to induce swirling flow. The vortical flow due to roll is symmetrical with respect to the tank centerline, while those induced by pitch and yaw maneuverings showed two vortices side by side. The study found that without the anti-vortex baffle, the swirling flow caused surface dip during the late stage of drainage and hence early vapor ingestion. The flow can also be non-uniform in the drainage pipe as the secondary swirling flow velocity component can be as high as $10 \%$ of the draining velocity. An analysis of the vortex dynamics shows that the swirling flow in the drainage pipe during the Upper Stage burn is mainly the result of residual vortices inside the tank due to conservation of angular momentum. The study demonstrated that the swirling flow in the drainage pipe can be effectively suppressed by employing the anti-vortex baffle.
\end{abstract}

\section{INTRODUCTION}

Many space and launch vehicles generally use a liquid propellant stored in storage tanks and fed into engines during launch and maneuvering in space. The liquid must be moved from 
the storage tanks to the engine in an efficient manner. First, vapor or gas cannot be allowed to enter the engines in any great amount or too early during the ignition process. If gas is introduced into the engines, it may cause a stall or other malfunctioning of the engine that may increase the possibility of engine failure. The conventional approach is to place a device in the propellant storage tanks to reduce the acquisition of gas into the engine. Second, it is desirable to completely empty the storage tanks during an engine burn and during flight to reduce re-entry weight and increase vehicle payload. Typically, a portion of liquid propellant remains in the tank, thereby increasing the vehicle weight and reducing the maximum allowed payload. At the NASA Ares I program Upper Stage (US) Main Propulsion System (MPS) Preliminary Design Review, two issues of major concern were identified to be a) the vehicle dynamics and b) ullage pressure collapse due to propellant sloshing. CFD analyses have been performed [1] to assess the $1^{\text {st }}$ and $2^{\text {nd }}$ mode stability limits for individual axial, side and oscillatory loads. During the actual Crew Lunch Vehicle (CLV) ascent, however, sloshing is directly related to the vehicle induced body force which is a summation of three sources: vehicle linear acceleration with respect to its mass center, vehicle maneuvers (roll, pitch, and yaw), and thrust oscillations (TO). The purpose of this study is to derive extra forces in the non-inertial frame due to maneuvers, to implement the body forces into an existing free surface CFD code, CFD-ACE+, and to assess the effect of the predicted vehicle body forces on the formation of vortical flow during Ares I First Stage (FS) burn, FS separation, and US burn.

\section{RESULTS AND DISCUSSION}

\section{COMPUTATIONAL MODEL}

During the CLV flight, the vehicle dynamics are influenced by: a) vehicle acceleration on the center of gravity; and b) vehicle maneuvers of roll, pitch and yaw. The three dimensional CFD models for the full scale Ares I Upper Stage LH2 and LOX tanks are shown in Figure 1. 


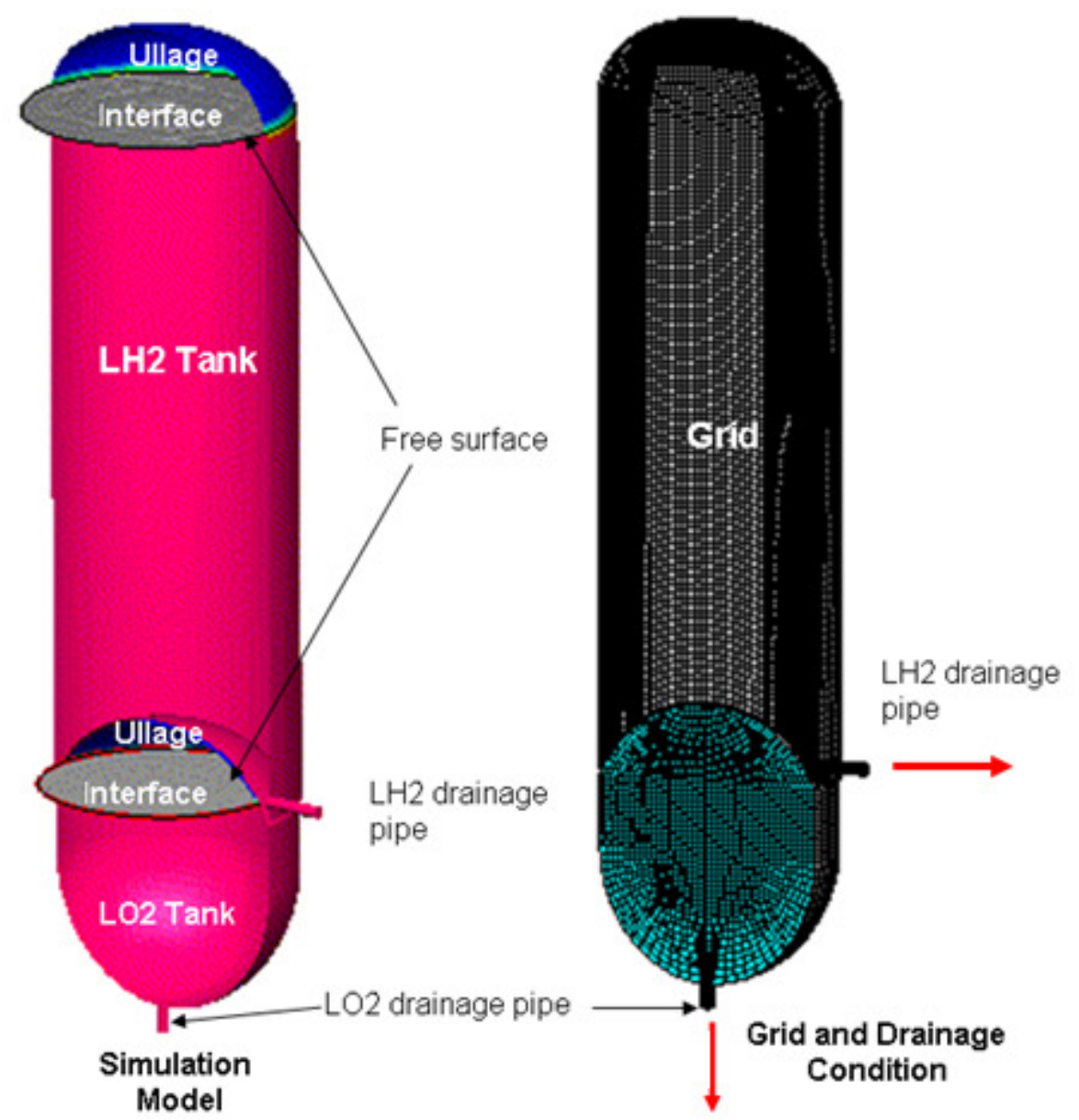

Figure 1. Computational model and grid for the Ares I Upper Stage propellant tanks

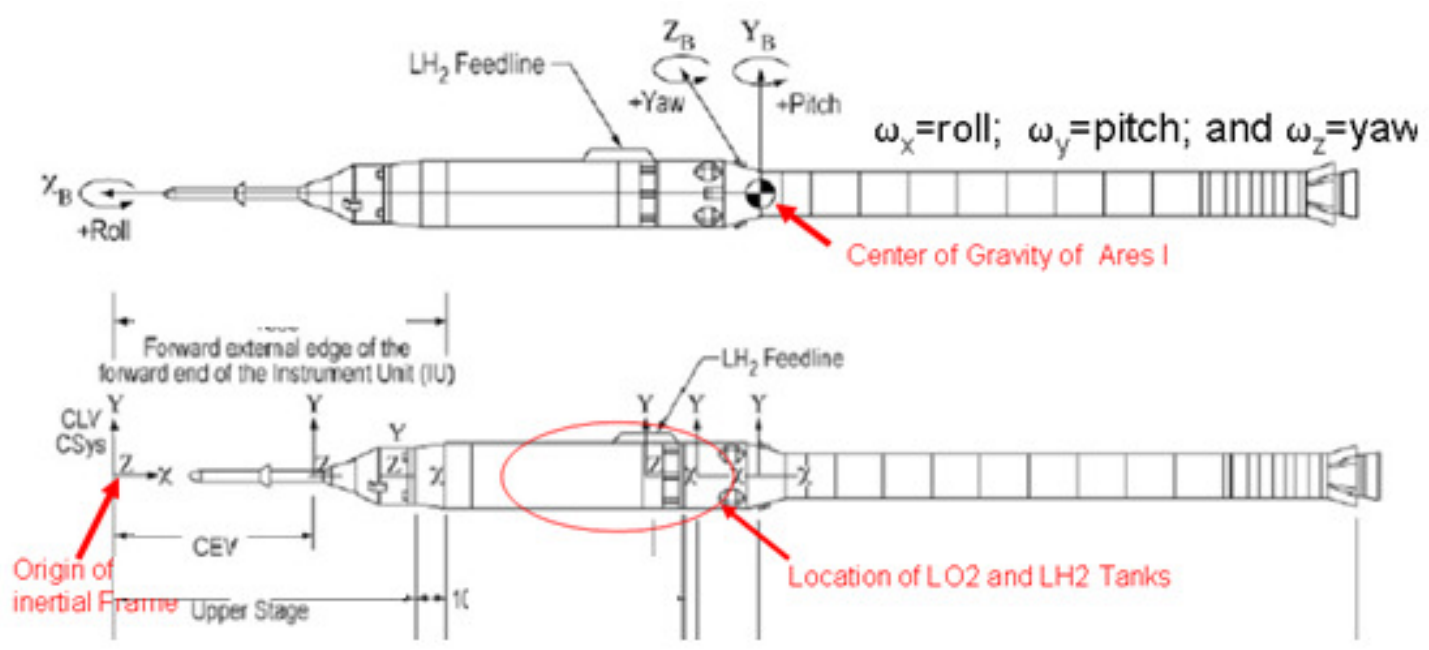

Figure 2. Crew Lunch Vehicle (CLV) Control Body Coordinate System

Since the tanks are subjected to the body forces in all three dimensions, the entire geometry of the tanks is included in the simulation model. Extra drainage pipes are built-in to simulate the draining during the US burn. The coordinates of the computational grid are located at the same position as the CLV control body coordinates system of Figure 2 . The details of the anti- 
vortex baffle model for the LOX tank are shown in Figure 3. Here the baffle is modeled as a solid pie-sector with a thickness of 2 degrees in the circumferential direction. For the LOX tank with the baffle, there is a total of 223,000 cells, and for the LOX tank without the baffle, the cell count reduces to 50,212 . There are at least 38 cells across the tank diameter. This ensures that the first natural mode is resolved by at least 76 cells, and the second natural mode is resolved by at least 38 cells. An O-grid is used to align the grid surface with the baffle surface. The initial condition is a quiescent flow, and the positions of the free surface are as shown in Figure 2.

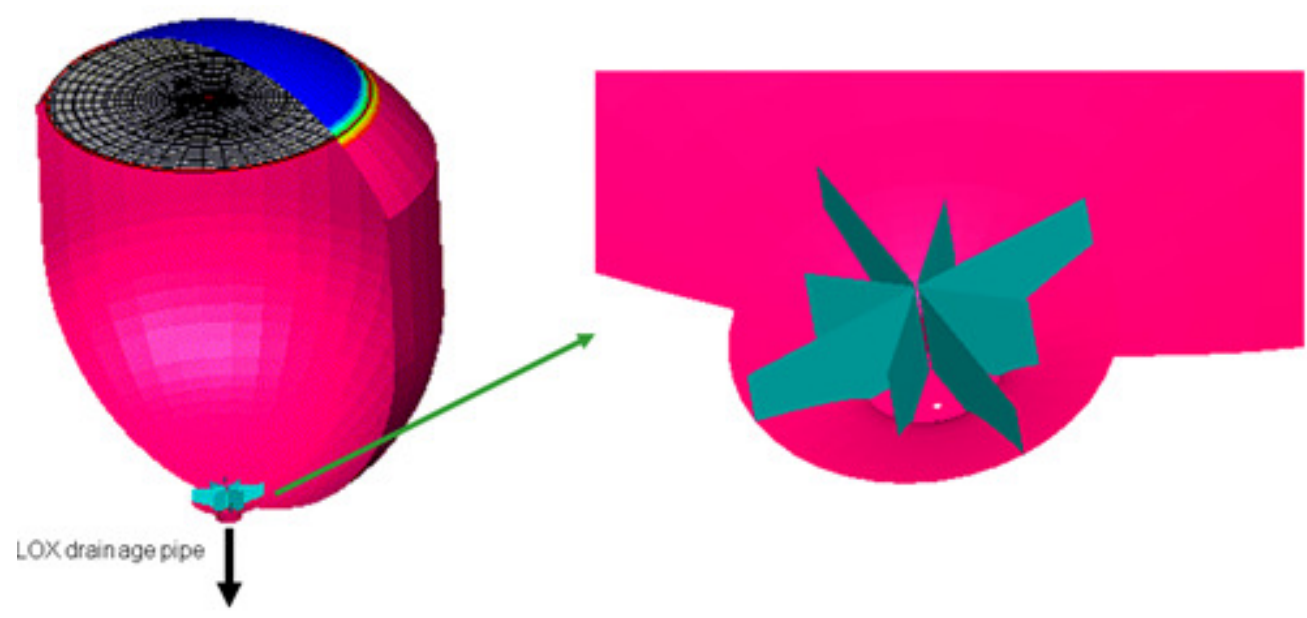

Figure 3. Computational model and grid for the Ares I LOX tank with anti-vortex baffle

In the computational model, both gas and liquid phases are assumed to be incompressible. The physical properties of both phases, such as density, viscosity, and surface tension, are taken as constant. The flow is assumed to be laminar without use of any turbulence model. When the US engine starts, the drainage pipe exit is given a constant mass flow rate boundary condition.

\section{SLOSH AND VORTICAL FLOW ANALYSIS FOR LOX TANK WITHOUT ANTI-VORTEX BAFFLES}

\section{First Stage Burn}

The case without the anti vortex baffle is presented first to gain insight into the generation and transport of vortical flow inside the LOX tank. During the first stage flight, there is no mass flow out of the tank, and flow motion inside tank is in response to the maneuvering forces. To better understand the contribution of various body forces, Figure 4 shows the non-dimensional angular accelerations by taking the temporal derivative of the angular velocities. From a back-ofthe-envelope calculation, it is estimated that the non-dimensional centrifugal force, which is proportional to the square of the rotational speed, is rather negligible as it is on the order of $1.0 \times 10^{-4}$. On the other hand, the contribution of angular acceleration is rather significant, as in addition to the long moment arm as discussed above, there are several peaks of the nondimensional angular acceleration visible from Figure 4. For example one can see both pitch and yaw maneuvers around non-dimensional time 6 after the lift-off. At these peaks (in very short time), the resulting side loads are as high as $0.3 \mathrm{~g}$. These accelerations in pitch and yaw can drive the first mode slosh inside the tanks. Free surface altitude plotted in Figure 8 is an indication of the instantaneous slosh dynamics inside the tank. As the magnitudes of pitch and yaw are about the same, the axis of the first slosh mode is aligned at 135 degrees to the $y-z$ axes, as evident in Figure 4. The above lateral slosh induces vortical flow inside the drainage pipe in the form of two vortices side by side, also shown in Figure 4. 


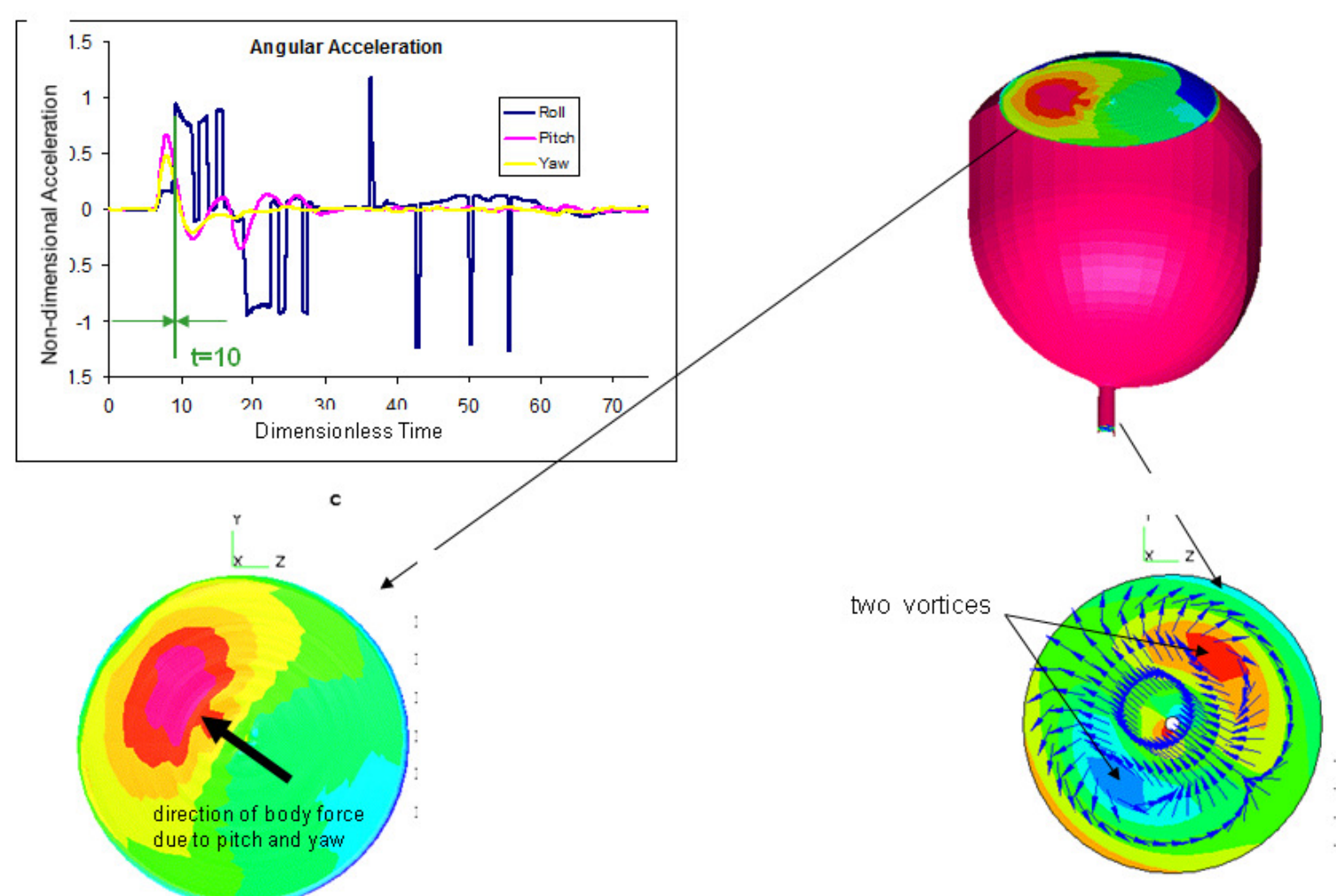

Figure 4. Slosh and vortical flows induced by maneuvering during the first stage burn

\section{STAGE SEPARATION}

Next, the flow dynamics during stage separation are considered. The stage separation includes burn down of the FS motor, then separation where the environment is briefly low $\mathrm{g}$ $(0.01 \mathrm{~g})$, followed by a sudden $0.1 \mathrm{~g}$ impulse due to the ullage rocket firing, and finally a gradual thrust build-up from the US engine. During this time frame, the vehicle experiences maneuvers that cause several spikes in angular accelerations (see Figure 5), and these large changes in roll and pitch rates lead to the first mode slosh and spinning mode (rotary mode [4]) in the tanks. In the meantime, the sudden drop in vertical acceleration (see Figure 5) from $4 \mathrm{~g}$ to $0.01 \mathrm{~g}$ amplifies the sloshing motion. The present simulation results did not show any instability of the surface during the stage separation, but one can see slow slosh motion during stage separation. This slow down in the motion of both the first and spinning modes is due to the reduced natural frequency $f$. Based on the dispersion relation of the tank sloshing frequency [1], $f$ is related to $g$ as:

$$
f=\sqrt{\frac{g}{2 \pi \lambda}}
$$

where $\lambda$ is the wavelength of the slosh mode. For the first mode, its wavelength is twice the tank diameter. During the stage separation as the vertical acceleration varies from $4 \mathrm{~g}$ to 0.01 $g$ (see Figure 5), the natural frequency of the first slosh mode of the tank reduces 20 times. This reduction slows down the surface motion. 


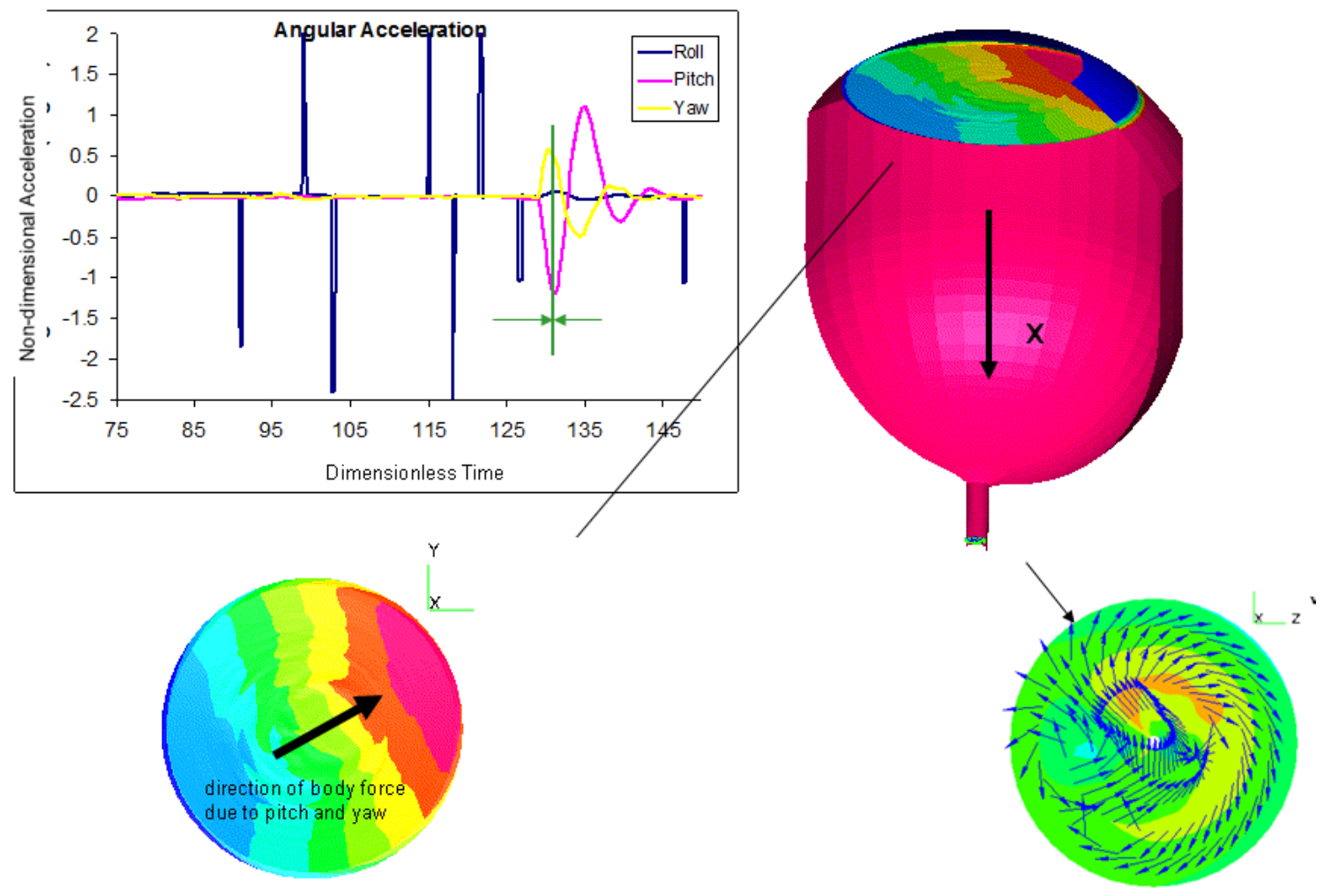

Figure 5. Slosh and vortical flows induced by maneuvering during stage separation

To better understand the vortical flow inside the drainage pipe, two types of the vortical flows induced by maneuvering vehicle are shown in Figure 6 . Swirling flow induced by vehicle roll, which is symmetric to the center of the tank axis, is shown on the left, and vortical flow induced by vehicle yaw and pitch, which has two vortices side by side, is shown on the right. The vortical flow in the drainage pipe is expected to exhibit a combination of the characteristics observed from the above two types of the flow.

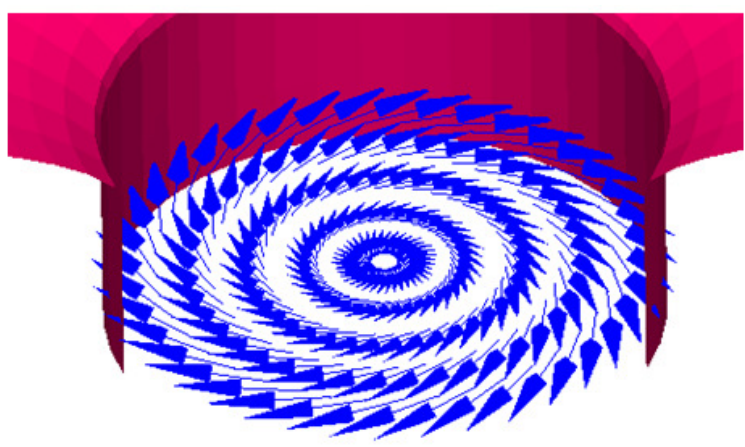

Swirling flow due to rolling: symmetrical to the center of the tank axis

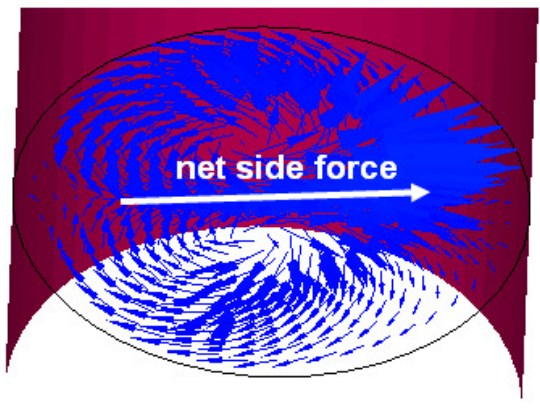

Swirling flow due to side forces: two vortices side by side

Figure 6. Two types of vortical flows inside drainage pipe: one induced by rolling and one by pitch and yaw

\section{UPPER STAGE BURN}


During the second stage burn, the liquid flows out of the LOX tank drainage pipe to supply the fuel and oxidizer. The maneuver as shown in Figure 7, consists of mainly the multiple alternating peaks in roll acceleration, while the variation in pitch and yaw accelerations are much smaller. The cross-sectional flow velocity inside the pipe at different dimensionless time instances is shown in Figure 11. Even though there is a continuous change in the sign of roll acceleration during the upstage burn, the rotation sense of the swirling flow inside the pipe is always the same. This implies that the vortices in the drainage pipe during the US burn come from the residual vortices in the tank. The original vortices inside the tank may be small but they are amplified while going through the convergent bottom section of the tank and the pipe due to conservation of angular momentum. To explore this, one can consider the vorticity transport equation in the form of:

$$
\frac{D \vec{\omega}}{D t}=v \nabla^{2} \vec{\omega}+\vec{\omega} \bullet \nabla \vec{V}
$$

The above equation is obtained by taking curl of the Navier-Stokes equation and assuming incompressible flow. The left hand side term and the first term on the right hand side are the typical transport equation for a scalar with convection and diffusion, while the last term represents stretching or tilting of vorticity. It is this term that contributes to the strengthening of vorticity as the flow passes through the drainage pipe.

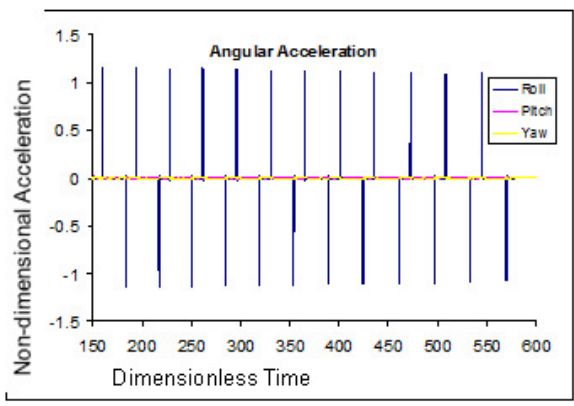

Less pitch andyaw, more roll spikes

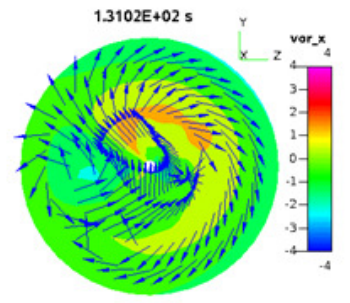

Dimensionless $\mathrm{t}=131$

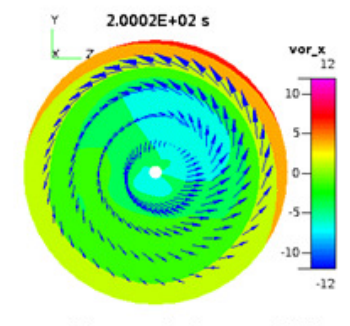

Dimensionless $\mathrm{t}=200$

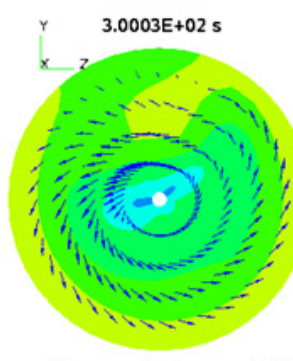

Dimen sionless $\mathrm{t}=300$

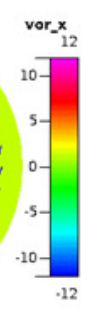

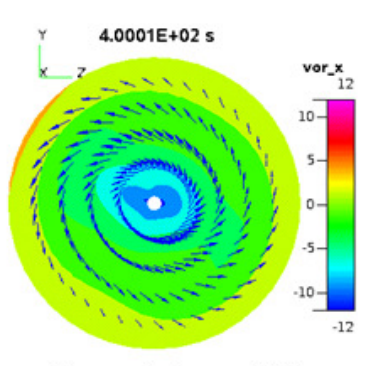

Dimensionless $\mathrm{t}=400$

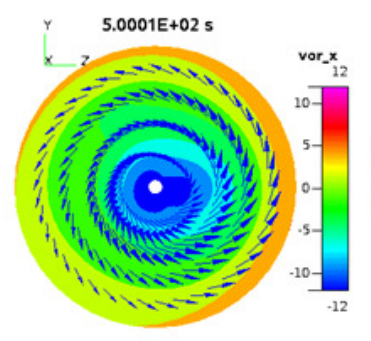

Dimensionless $\mathrm{t}=500$

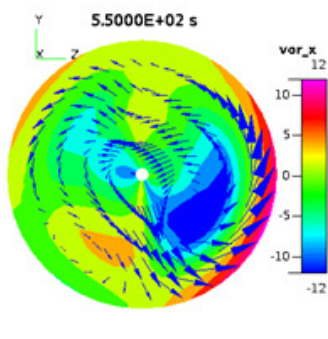

Dimensionless $\mathrm{t}=550$

Figure 7. Slosh and vortical flows induced by maneuvering during the second stage burn.

For the LOX tank with the coordinates as shown in Figure 8, let us consider the $\mathrm{x}$ component of vorticity:

$$
\frac{D \omega_{x}}{D t}=v \nabla^{2} \omega_{x}+\omega_{x} \frac{\partial v_{x}}{\partial x}
$$

The bottom half of the LOX tank is like a convergent pipe, where the area decreases with $x$. Since the total outflow mass is constant, the derivative of $v_{x}$ with respect to $x$ is positive, meaning that there is vorticity source present in the equation. In a simple sense, this represents 
the conservation of angular momentum. A spinning ice skater is a common example of conservation of angular momentum. When the skater starts spinning with hands outstretched, the angular velocity is low, but the spinning becomes very fast as the hands are pulled in. This is because the angular velocity increases as the moment of inertia decreases in order to conserve angular momentum. It is due to this angular momentum conservation that the vorticity increases as the flow drains into the pipe. Figure 8 gives the cross-sectional velocity at a given instance at different axial $(x)$ locations.

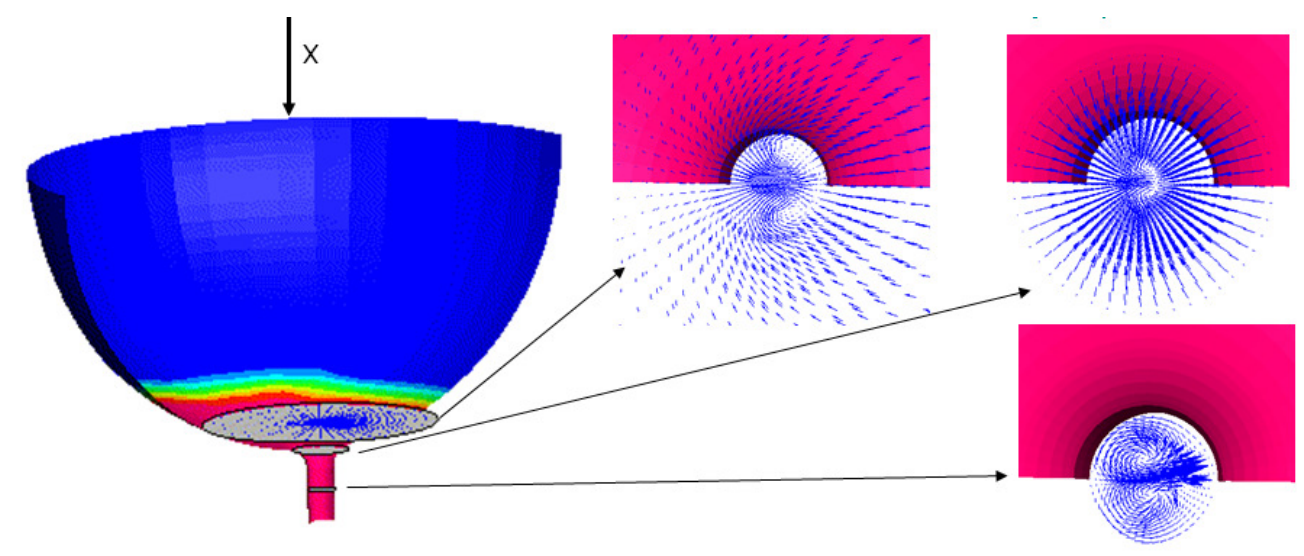

Figure 8. Transport physics of a vortex on the LOX tank during draining. Notice the increase in the vorticity along the flow path.

\section{IMPACT OF SWIRLING FLOW}

As found from the above investigation, without an anti-vortex baffle, there is a significant swirling flow during the draining of the tank. The swirling flow can lead to non uniformity in the flow velocity as illustrated in Figure 9, which shows the flow path of a group of particles as they travel through the pipe. The magnitude in the circumferential velocity is found to be as high as $10 \%$ of the mean drainage flow velocity. The swirling flow also causes early gas ingestion into the engine and leads to higher residual mass as evident in Figure 10. The design consideration is that the liquid must be moved from the propellant tanks to the engine in an efficient manner. First, vapor or gas cannot be allowed to enter the engines in any great amount or too early in the ignition process. If gas is introduced into the engines, it may cause a stall or other malfunctioning of the engine that may increase the possibility of engine failure.

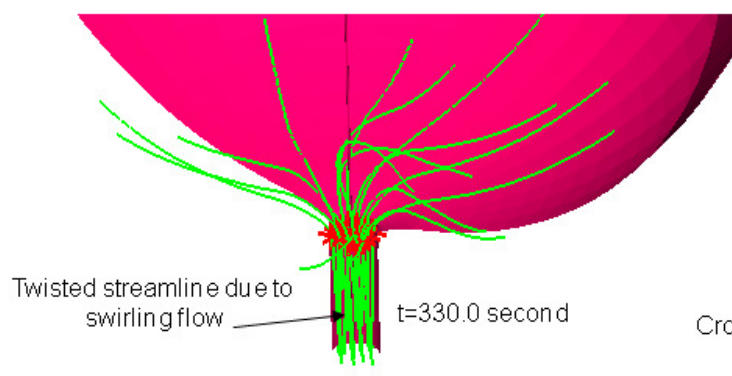

Cross-section al velocity vector and vorticity

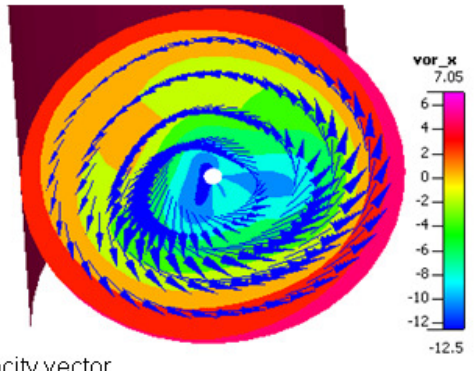
$\mathrm{t}=330.0$ second

Figure 9. Non-uniform flow into turbo pump due to vortical flow at dimensionless time of 300 second. 

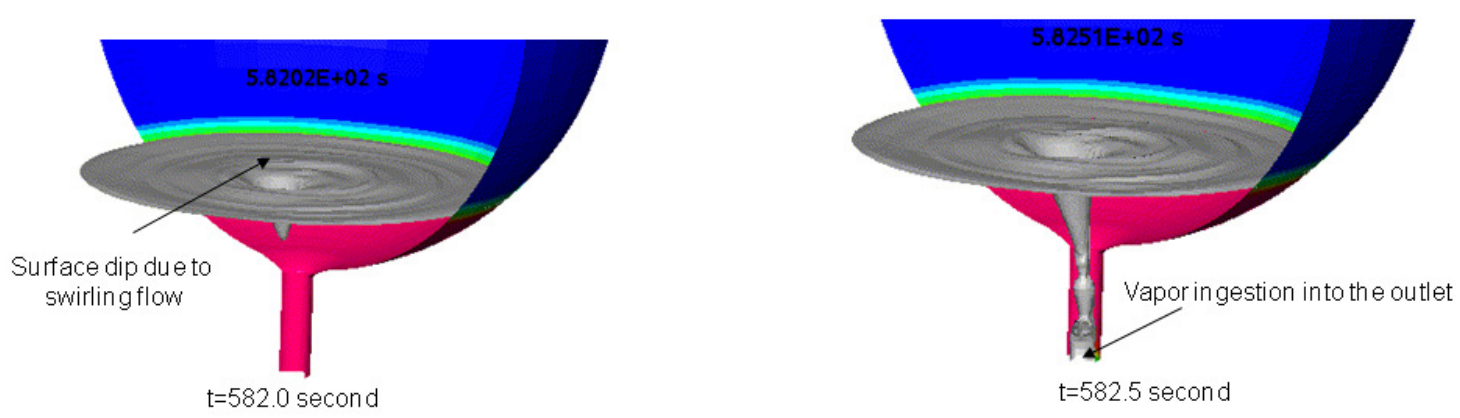

Figure 10. Formation of a dip and earlier vapor ingestion into the outlet, leading to higher residual mass

To have a better understanding of the impact of swirling flow on the surface dip and early vapor ingestion, one should notice that swirling flow induces a centrifugal body force in the tank, which is proportional to $\rho \omega^{2}$. This body force gives rise a pressure gradient which is in the form of:

$$
\nabla p=\rho r \omega^{2}
$$

The pressure increases with radius $r$. During the early stage of draining, this pressure gradient is relatively small, however at the later stage, the pressure gradient amplifies due to conservation of angular momentum:

$\nabla p=\rho \omega^{2} r=\rho \frac{(r \omega)^{2}}{r}=\rho \frac{(\text { Const } .)^{2}}{r}$

When $r$ is small, there is a rapid pressure drop at the center of the interface causing the dip and early vapor ingestion.

\section{SLOSH AND VORTICAL FLOW ANALYSIS FOR LOX TANK WITH ANTI-VORTEX BAFFLES}

The above study indicates that without the anti-vortex baffle, the swirling flow causes surface dip during the late stage of drainage and hence early vapor ingestion. The flow is also non-uniform in the drainage pipe as the secondary swirling flow velocity component can be as high as $10 \%$ of the draining velocity. In the design of US engine, the consideration is that in addition to the requirement that the liquid must be moved from the storage tanks to the engine in an efficient manner, vapor or gas cannot be allowed to enter the engines in any great amount or too early in the ignition process. If gas is introduced into the engines, it may cause a stall or other malfunctioning of the engine that may increase the possibility of engine failure. Another requirement is to empty the propellant tanks as completely as possible during an engine burn and during flight to reduce re-entry weight and increase vehicle payload. Typically, a portion of liquid propellant still remains in the tank, thereby increasing the vehicle weight and reducing the maximum payload of the vehicle. The swirling flow in the LOX tank has been shown in causing high residual mass in the tank. It is general practice to place a device, such as an anti-vortex baffle, to substantially reduce or eliminate the formation of the dip and vortex, as well as to prevent vapor ingestion into the outlet as the liquid drains out during the flight. A typical antivortex baffle is shown in Figure 11, and its computational model has been developed in this study. The features of the resulting flow dynamics are discussed below. 


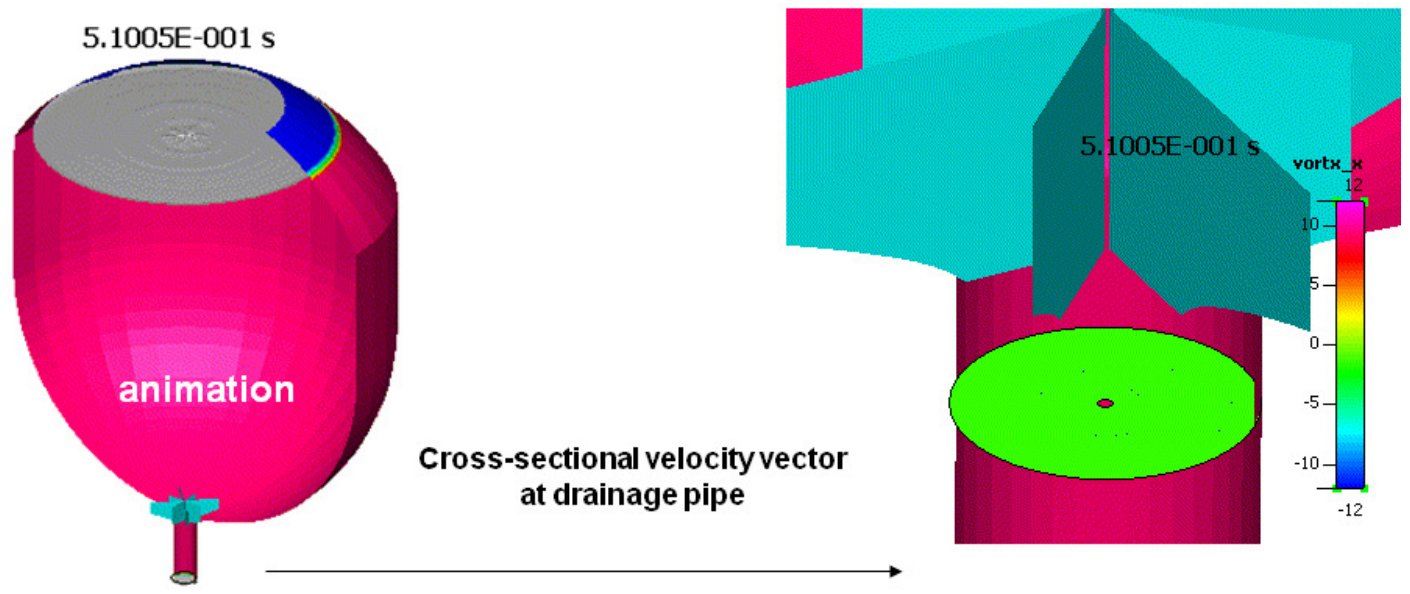

Figure 11. Vortical flow modeling with anti-vortex baffle

\section{FIRST STAGE BURN AND STAGE SEPARATION}

One can see from Figure 11 that the anti-vortex is located above the drainage pipe, and there is no flow passing through the baffle before the start of Upper Stage. It is expected from the fluid dynamics point of view that the cross-section flow of the drainage pipe is the same as the case without the baffle. This is indeed the situation observed in the present simulation, so we will concentrate on the second stage burn. 


\section{UPPER STAGE BURN}

With the presence of the anti-vortex baffle, the dynamics of the flow has changed significantly. Figure 12 shows the particle traces with and without the baffle assembly. Due to the swirling flow in the case without the baffle, the 3D particle trace from the pipe inlet shows circumferential motion due to the swirling flow, and the top view of the trace shows the direction and strength of the cross flow, which is high as $10 \%$ of the main flow. On the other hand, with the use of anti-vortex baffle, the swirling component has been eliminated, and only radial motion of the particles is observed. The illustration of the velocity vectors in Figure 12 further verifies the effectiveness of the anti-vortex baffle.

The effectiveness of anti-vortex baffle in reducing the residual mass is demonstrated in Figure 13. With the use of the baffle the surface dip has been eliminated before the vortical flow reaches the baffle assembly, and one can see very small amount of the residual mass at the time of vapor ingestion (Figure 14).
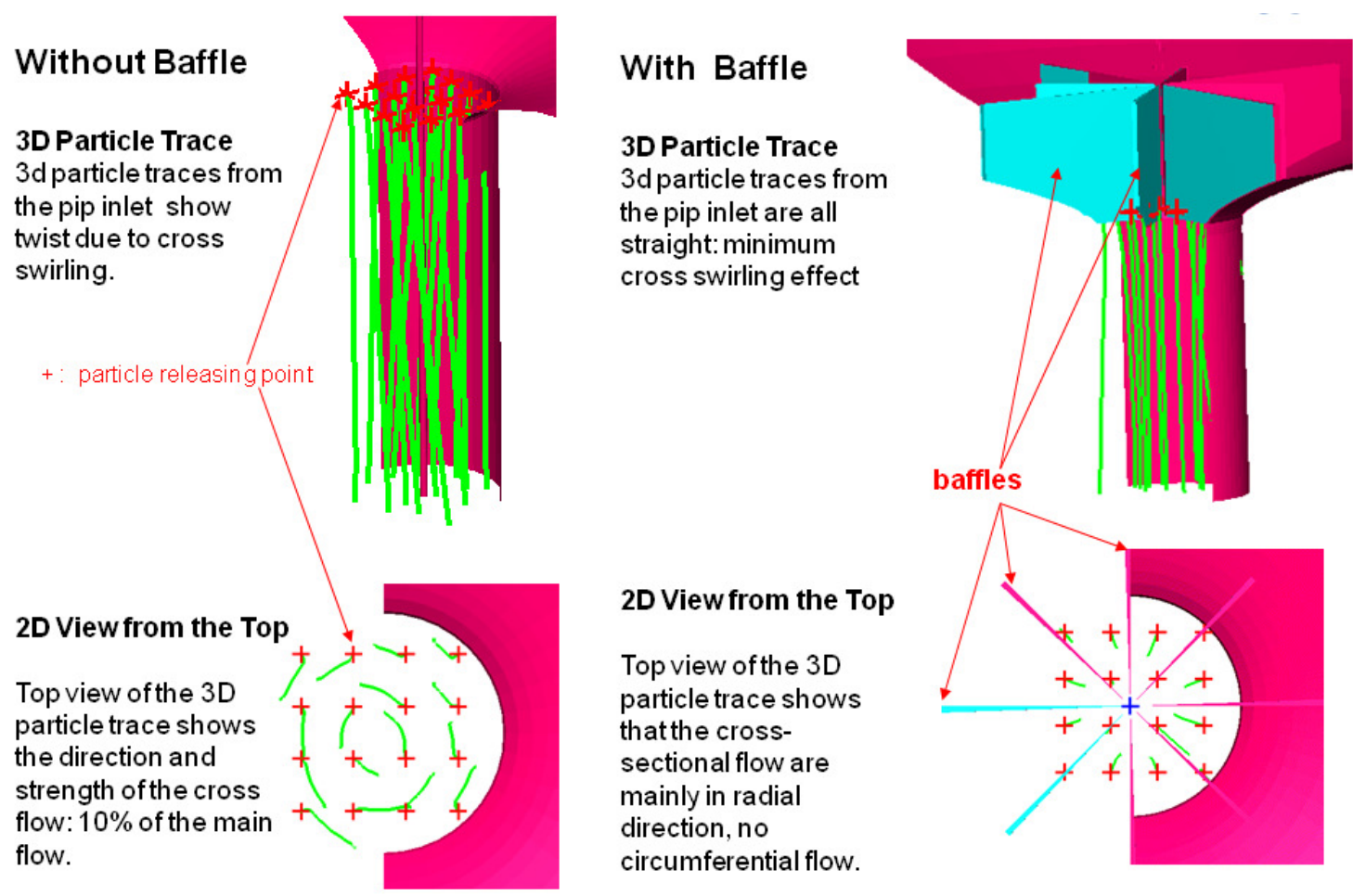

Figure 12. 3D particle traces near the entrance of the pipe for the cases with and without antivortex baffle. 

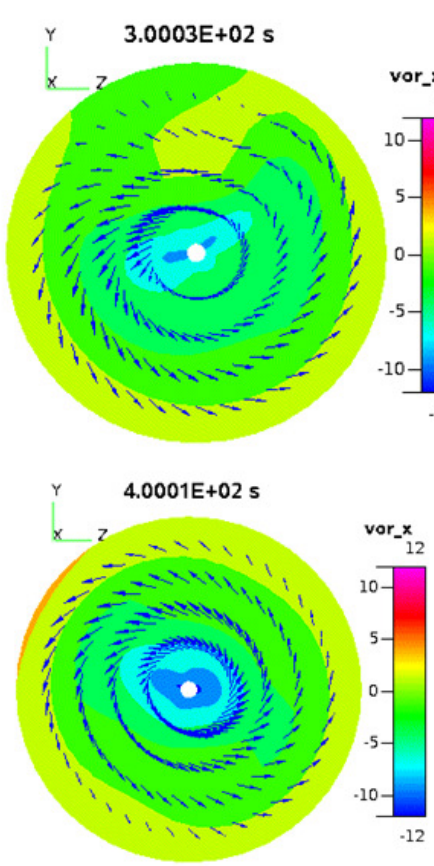

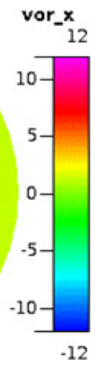

$\longleftarrow$ Without Baffle

(a) $t=300 \mathrm{~s}$

$\longleftarrow$ Without Baffle

With Baffles

(b) $\mathrm{t}=400 \mathrm{~s}$
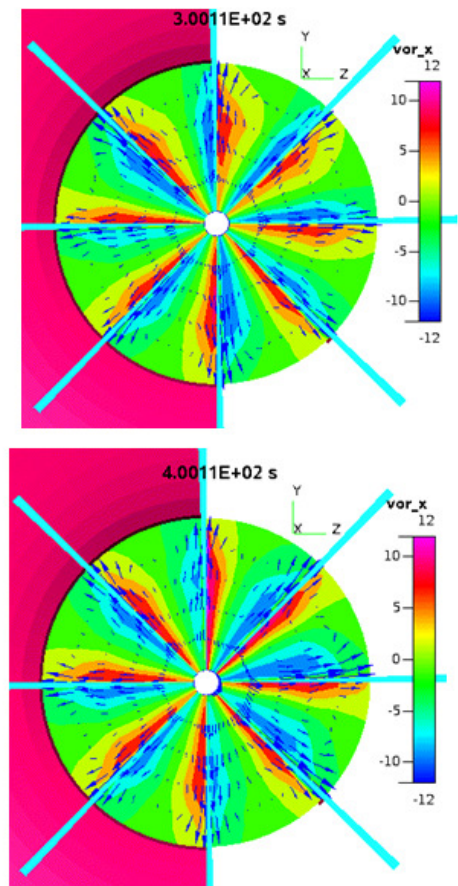

Figure 13. Cross-sectional velocity vectors for the cases with and without anti-vortex baffle at dimensionless time of 300 and 400.
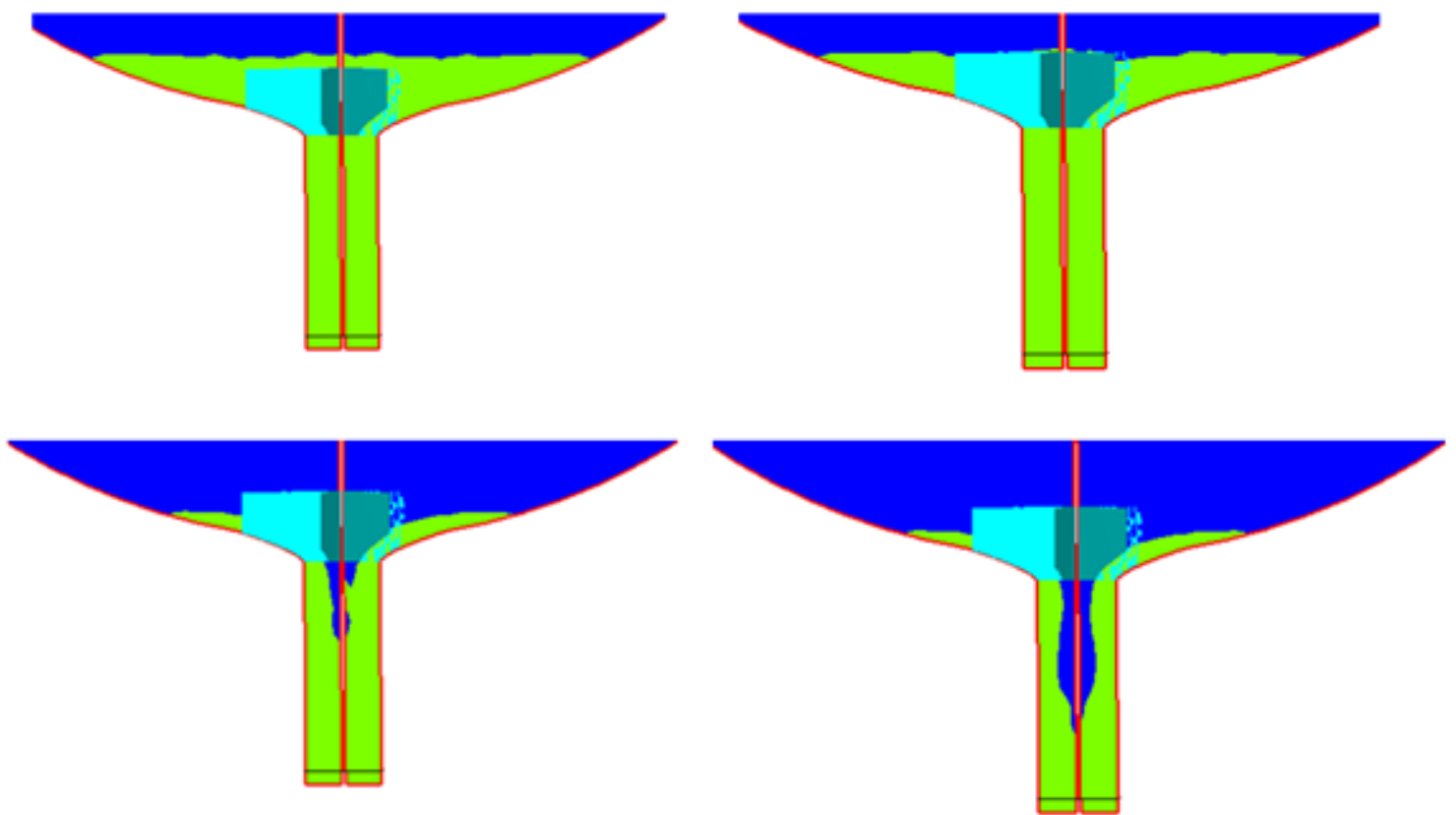

Figure 14. Residual mass during the liquid draining at the later stage of US burn. 


\section{SUMMARY AND CONCLUSIONS}

The Navier-Stokes equations in the non-inertial frame are used to study the vortical flow sources during full trajectory launch vehicle ascent. The body forces from Six Degree-OfFreedom (6-DOF) dynamics predicted by GN\&C analysis were used as input to the CFD code. Analyses were performed for the cases of the LOX tank with and without the anti-vortex baffle during CLV flight from earth to orbit. Due to the large moment arms, the vehicle maneuvers are found to have significant impact on vortical flow source. The side loading due to pitch and yaw maneuverings leads to the formation of a first mode slosh wave and two vortices side by side. Roll maneuvering induces spinning motion of the liquid surfaces, which in turn forms vortices during the tank draining. Due to conservation of angular momentum, the residual vortices in the LOX tank are shown to be amplified during the second stage burn. The study found that without the anti-vortex baffle, the swirling flow caused surface dip during the late stage of drainage and hence early vapor ingestion. The flow can also be non-uniform in the drainage pipe as the secondary swirling flow velocity component is as high as $10 \%$ of the draining velocity. The study demonstrated that the swirling flows in the drainage pipe can be effectively suppressed by utilizing the anti-vortex baffle. As a result, the flow uniformity in the pipe is improved, and the residual mass is significantly reduced.

\section{ACKNOWLEDGEMENTS}

This study was performed under a Task Order of the Jacobs Engineering NASA MSFC ESTS Contract NNM05AB50C. Dr. Ram Ramachandran was the Jacobs ESTS Task Lead, and Dr. Jeff West (MSFC Fluid Dynamics Branch, ER42) was the NASA MSFC Task Monitor.

\section{REFERENCES}

1.H. Q. Yang, J. M. Rojahn, J. W. Peugeot, D. J. Dorney. CFD Analysis of Propellant Tank sloshing under Vertical Oscillatory Thrust and Horizontal Oscillatory Side Load, JANNAF 2008, Paper LPS-06, Feed System Design and Analysis, Orlando, November 10-13, (2008). 2.H. Q. Yang and J. W. Peugeot, Propellant Sloshing Parameter Extraction from CFD

Analysis, 46th AIAA/ASME/ASE/ASEE Joint Propulsion Conference \& Exhibit, July 25-28 2010, Nashville, TN. AIAA 2010-6889.

3.H. Q. Yang and J. W. Peugeot, Surface Instability of Liquid Propellant under Veritical Oscillatory Forcing, 47th AIAA/ASME/ASE/ASEE Joint Propulsion Conference \& Exhibit, July 31-August 3,2011, San Diego, CA. AIAA 2011-5773.

4.D. D. Kana, A Model for Nonlinear Rotary Slosh in Propellant Tanks, J. of Spacecraft, Vol. 24, No. 2, pp. 169-177, 1987. 